\title{
Historical records of solar grand minima: a review
}

\author{
José M. Vaquero \\ Departamento de Física, Centro Universitario de Mérida, Universidad de Extremadura, \\ Avda. Santa Teresa de Jornet, 38, 06800, Mérida, Badajoz, Spain \\ email: jvaquero@unex.es
}

\begin{abstract}
Knowing solar activity during the past centuries is of great interest for many purposes. Historical documents can help us to know about the behaviour of the Sun during the last centuries. The observation of aurorae and naked-eye sunspots provides us with continuous information through the last few centuries that can be used to improve our knowledge of the long-term solar activity including solar Grand Minima. We have more or less detailed information on only one Grand minimum (the Maunder minimum in the second half of 17th century), which serves as an archetype for Grand minima in general. Telescopic sunspot records and measurements of solar diameter during Maunder minimum are available. In this contribution, I review some recent progress on these issues.
\end{abstract}

Keywords. Sun: activity, history and philosophy of astronomy.

\section{Introduction}

Historical documents can help us to know about the behaviour of the Sun during the last centuries including Grand Minima (Vaquero \& Vázquez 2009). On longer time scales, other procedures can also be used for the reconstruction of solar activity such as cosmogenic radionuclides (Usoskin 2008). There exists a wide range of possibilities to reconstruct solar activity on the basis of documentary sources (Figure 1). On the one hand, one has direct data, i.e. data obtained from observations of the Sun. And on the other hand, one has indirect data based on terrestrial phenomena linked to the behaviour of the Sun. Nonetheless, the indices that have been most used are those related to the observations of sunspots due to their versatility.

Some recent research on solar Grand Minima based on historical documents will be reviewed here. Therefore, the aim of this work is to show that this is a research field that can be fruitfully developed because there is interesting information that is still buried in archives and libraries.

\section{Naked-eye sunspot observations}

This section is devoted to naked-eye sunspot observations, and especially to their possible use for the reconstruction of solar Grand Minima during the last two millennia. It is occasionally possible to observe a sunspot by eye when it is sufficiently large and there are certain atmospheric conditions (mist, dust, smoke,...) which reduce the intensity of the Sun's light (see Vaquero \& Vázquez 2009, specially Chapter 2). The sunspot visibility problem should be approached as a problem of calculating contrast thresholds for the human eye (Schaefer 1991). Schaefer (1993) developed a theoretical model of sunspot visibility that can be applied to naked-eye observation. 


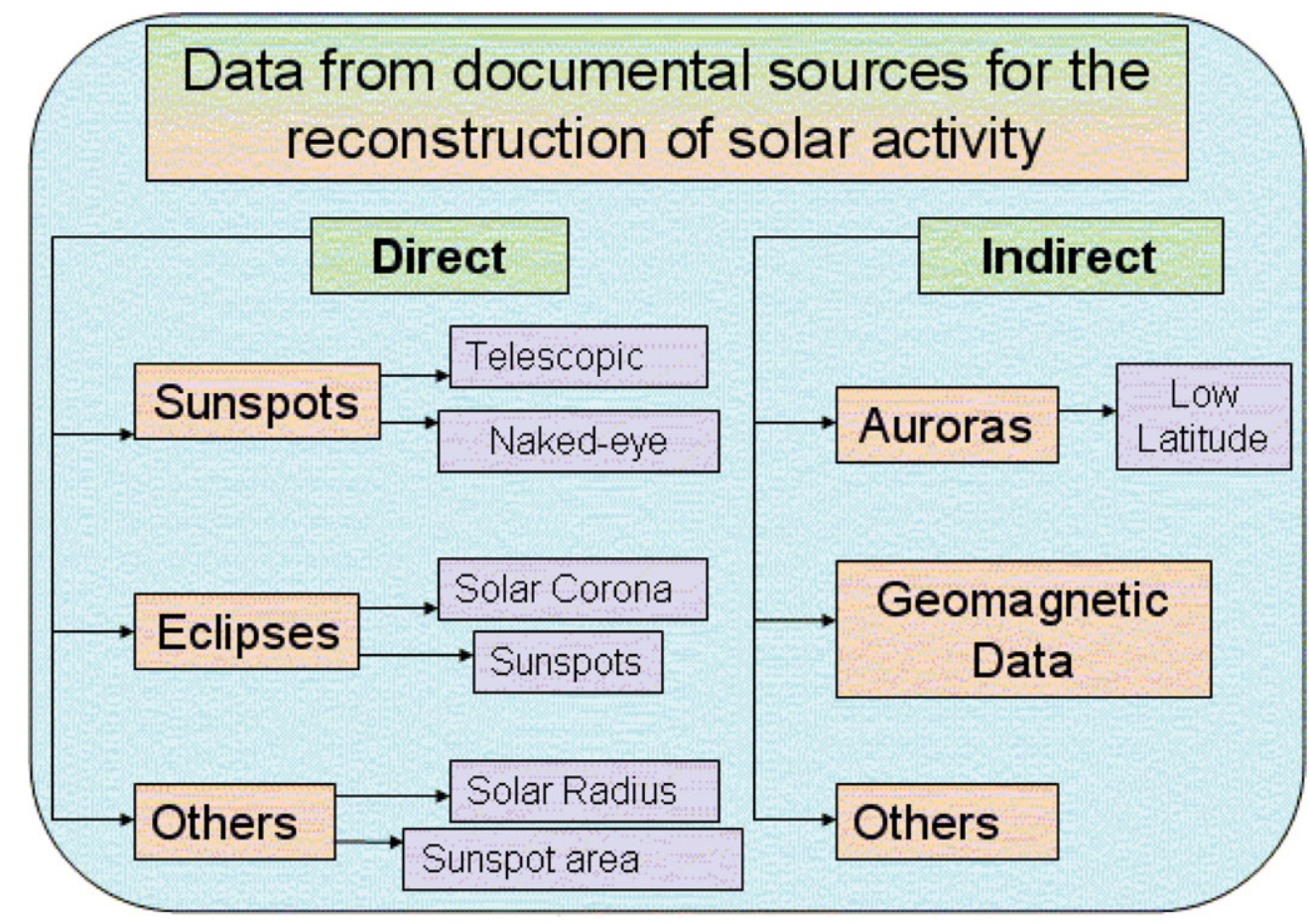

Figure 1. Different types of data from documentary sources for the reconstruction of solar activity.

A great number of historical naked eye sunspots are reported in Oriental historical sources (China, Korea, and Japan). Others are reported in European, Arabic (Vaquero \& Gallego 2002), Indian (Malville \& Singh 1995), and Maya (Burland 1958) sources. There are various catalogues of naked-eye sunspot observations available in the literature (Wittmann \& Xu 1987; Yau \& Stephenson 1988). In the last years, some new records appeared (Krivsky 1985; Stephenson \& Willis 1999; Vaquero \& Gallego 2002; Moore 2003; Lee et al. 2004; Vaquero 2004).

The reliability of naked-eye sunspot observations has been assessed by comparing carefully the Oriental sunspot sightings from 1862 onwards with contemporary Occidental white-light images of the Sun (Willis et al. 1996). These observations may be related to variations in some meteorological parameters (Willis et al. 1980; Eddy et al. 1989; Scuderi 1990; Hameed \& Gong 1991), such as the number of days of mist or dust storms. Nevertheless, some authors have reconstructed time series of annual numbers of naked-eye sunspots in spite of their evident problems (Nagovitsyn 2001 and Vaquero et al. 2002).

Vaquero \& Vázquez (2009) made a comparison between the solar activity reconstructed using cosmogenic radionuclides, the most reliable proxy to study the long-term behaviour of the Sun, and the series of naked-eye sunspot observations. Figure 2 shows the reconstruction of sunspot number made by Solanki et al. (2004) and a 50-year moving average of the annual number of naked-eye sunspot reconstructed by Vaquero et al. (2002) in arbitrary units. The naked-eye record is not useful for the study of long-term solar activity from $200 \mathrm{BC}$ to $\mathrm{AD} 800$ although some peaks of solar activity also appear in the naked-eye record. For the period AD 800-1600 approximately, the naked-eye sunspot record shows the most important periods of the solar activity although the amplitudes are variable. The two curves are quite similar during the period AD 1300-1600. Since the 


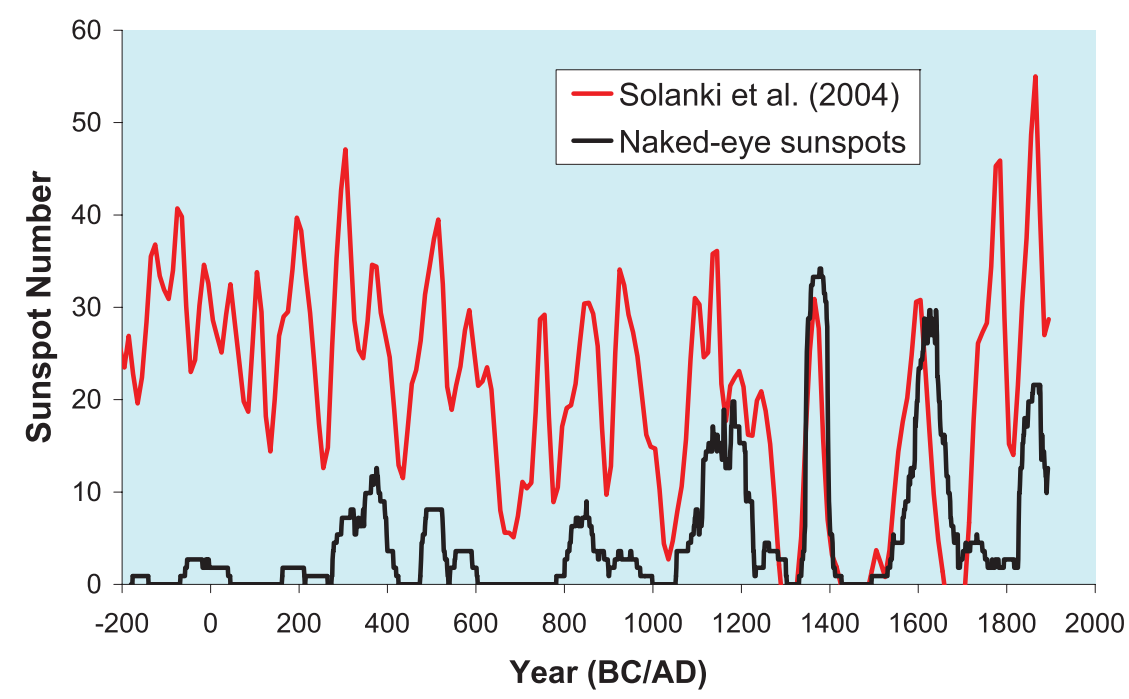

Figure 2. Reconstruction of sunspot number by Solanki et al. (2004) (red line) and using the 50 -year moving average of the annual number of naked-eye sunspots by Vaquero et al. (2002) (black line). Data were provided by I.G. Usoskin.

year 1600, the use of local sources in the Oriental record has made the correlation worse, and major differences can be observed during the period 1700-1825.

\section{Auroral observations in History}

Few phenomena have made as much of an impression on human beings as the aurora borealis, also known as the northern lights. The aurora is one of the most spectacular and earliest-known manifestations of the links between the Sun and the Earth. The northern and southern lights appear in the night sky with a great variety of colours and forms (Figure 3). The auroral activity is not rare, although few aurorae can be observed from low-latitude sites. Some general works on aurorae are Eather (1980), Brekke \& Eggeland (1994), Akasofu (2007), and Bone (2007).

The systematic study of the seasonal and secular variations of the auroral appearances needed data with perfectly dated and correctly compiled records of aurorae. Catalogues of aurora borealis were born of this need. The best-known catalogue of the 19th century was that of Hermann Fritz (1830-1893) who used many sources (Fritz, 1873). A catalogue of southern hemisphere aurorae was written by Boller (1898). Of the 20th century catalogues, we would cite as of special interest that written by Link $(1962,1964)$. Another useful catalogue was compiled by Krivsky \& Pejml (1988)Krivsky \& Pejml (1988) for the interval 1000-1900. They give dates of observation only and are restricted to latitudes $<55$ degrees.

Figure 4 shows the number of days per year (using a 5-year moving average and logarithmic scale) in which aurorae were recorded from the year AD 1000 to 1900 using the catalogue of Krivsky \& Pejml (1988). Very few aurorae before the Maunder minimum are recorded there, but the number increases considerably later. The number of auroral days per year during the first part of the total period is very low. During the 16th and 17 th centuries, this number begins to increase, although it is still small compared with the number of recorded auroral days during the 18th and 19th centuries. Note that the solar 


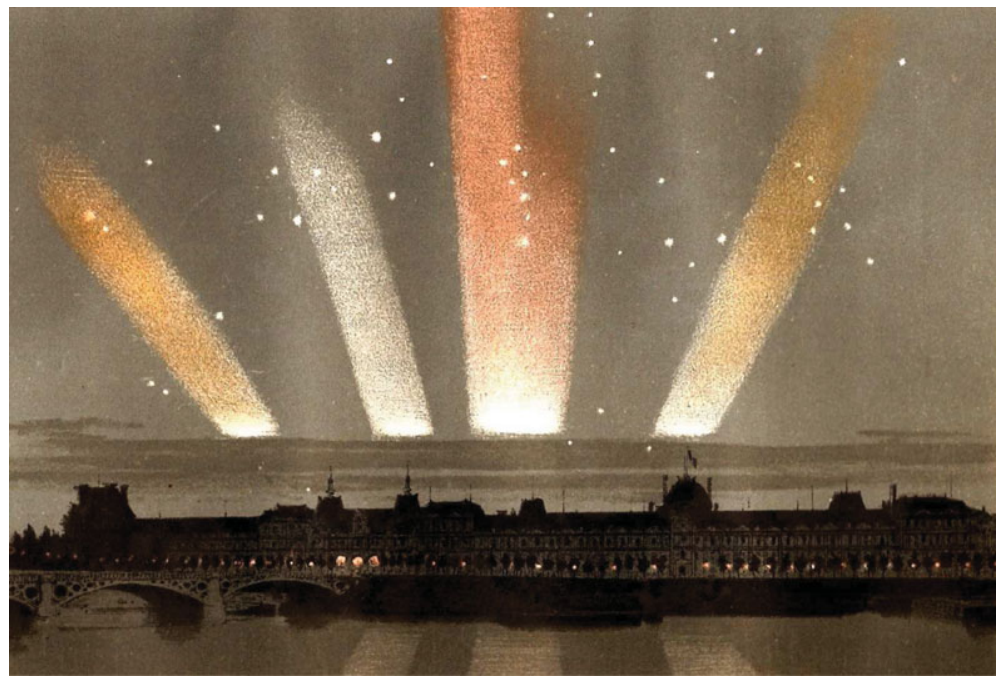

Figure 3. Sketch of the auroral display observed in Paris (13 May 1869) from Flammarion (1873).

Grand Minima of the last millennium appear clearly in this record in agreement with Solanki et al. (2004). Note also a minimum of auroral activity around 1765 (Silverman, 1992).

\section{Sunspot observations during the onset of Maunder minimum}

During the 400 years of systematic sunspot observations with telescopes (Hoyt \& Schatten 1998), several episodes of special interest have been recorded. Probably, the most interesting was the Maunder minimum (Eddy 1976, 1983; Ribes \& Nesme-Ribes 1993;

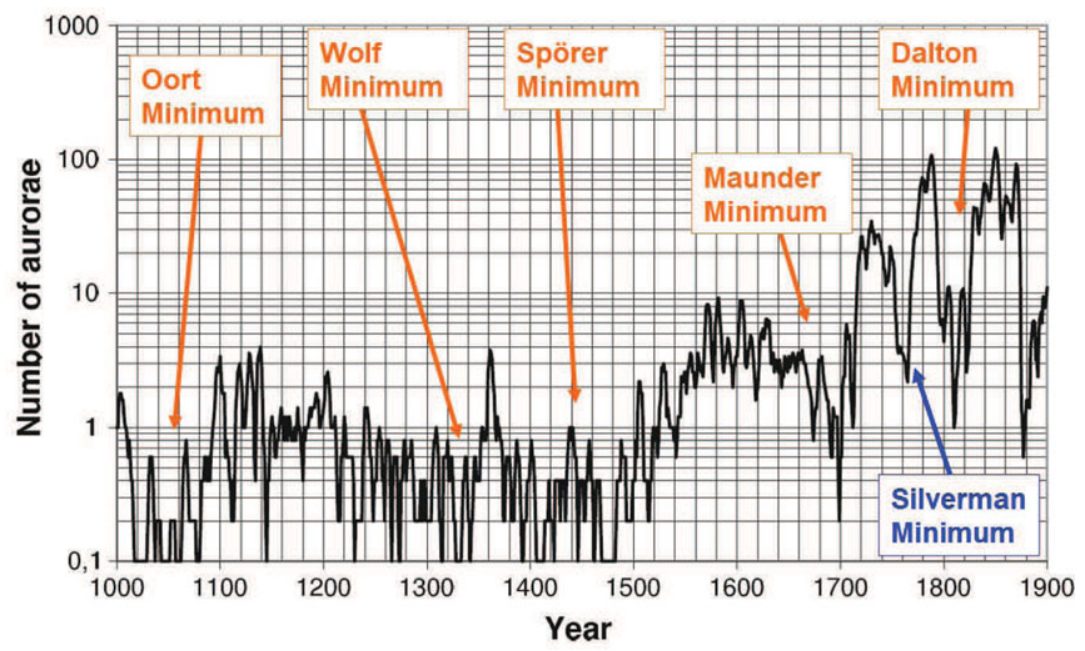

Figure 4. Smoothed annual number of auroral days (5-year moving average) recorded in the catalogue of Krivsky \& Pejml (1988). 


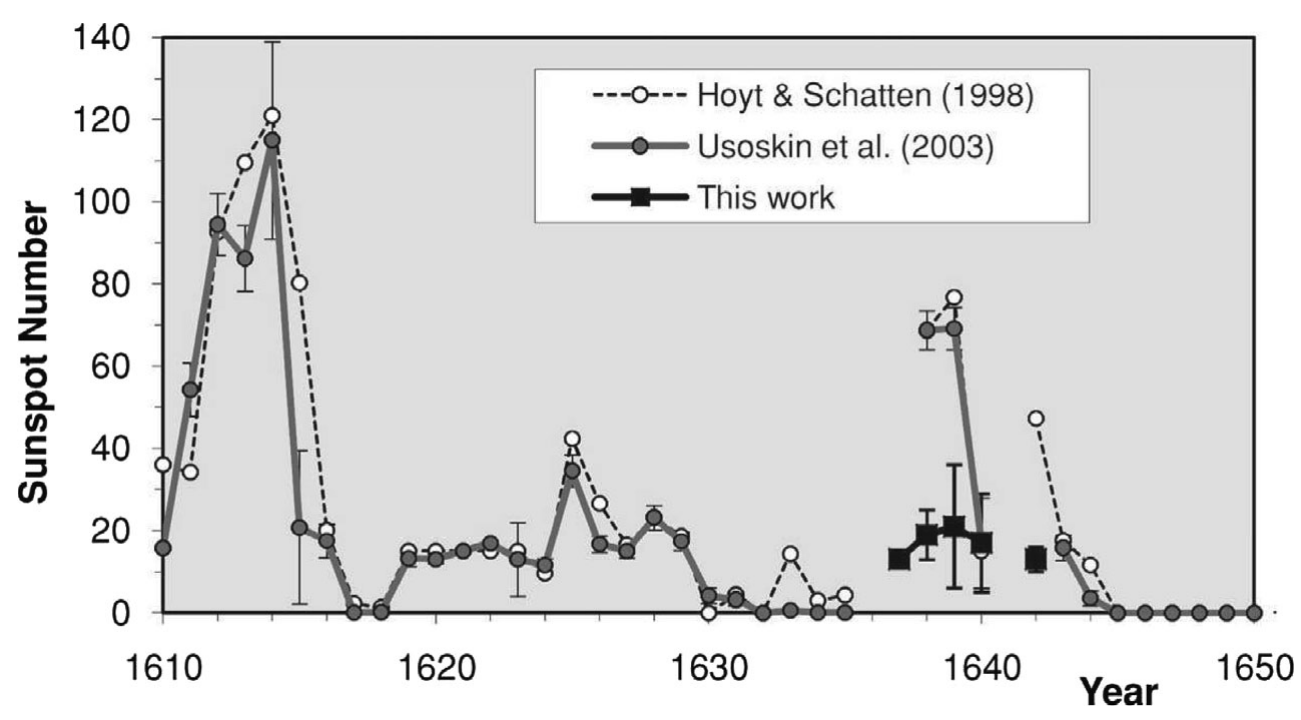

Figure 5. Annual sunspot numbers in the first half of 17 th century. Group sunspot numbers (Hoyt \& Schatten 1998) are shown by the dotted line, weighted sunspot number, based on the same data set (Usoskin et al. 2003) by the red line, and the weighted sunspot number estimated in Vaquero et al. (2011) by the black line.

Usoskin et al. 2001; Vaquero 2007). In the last few years, numerous studies have dealt with the Maunder minimum, including a review by Soon \& Yaskell (2003) and more recently by Miyahara et al. (2006). Another recent study considered solar rotation during the 17th century (Casas et al. 2006), using observations of a sunspot by Nicholas Bion.

The Maunder minimum forms an archetype for the Grand minima, and detailed knowledge of its temporal development has important consequences for the solar dynamo theory dealing with long-term solar activity evolution. The paradigm of the Grand minimum general scenario (Usoskin 2008) established that (i) transition from the normal activity to the deep minimum was sudden, (ii) a 22-year cycle was dominant in sunspot, and (iii) the recovery of the sunspot activity from the deep minimum to normal activity was gradual. Vaquero et al. (2011) reconsidered the paradigm of a Grand minimum general scenario by using newly recovered sunspot observations as well as revising some earlier uncertain data for the period 1636-1642, i.e., one solar cycle before the beginning of the Maunder minimum (Figure 5). The revised and updated data for 1637-1642 have essentially changed the profile of temporal variability of sunspot data before the Maunder minimum. In particular, the last solar cycle before the minimum now appears quite modest, with the peak value of 20 approximately compared with about 70 in the Group Sunspot Number series. This new scenario implies low solar activity roughly two solar cycles before the beginning of the Maunder minimum. Thus, transition from the normal activity to the deep minimum was not as sudden as previously thought. Figure 6 depicts a solar disk drawing by G. Marcgraf showing sunspots from 1637 September 21 to 25 as an example of historical documents used by Vaquero et al. (2011).

\section{Solar radius measurements during Maunder Minimum}

It is a matter of current debate whether the variations of the solar radius are correlated with the 11-year solar cycle. All the observations since 1650 have been compiled by 


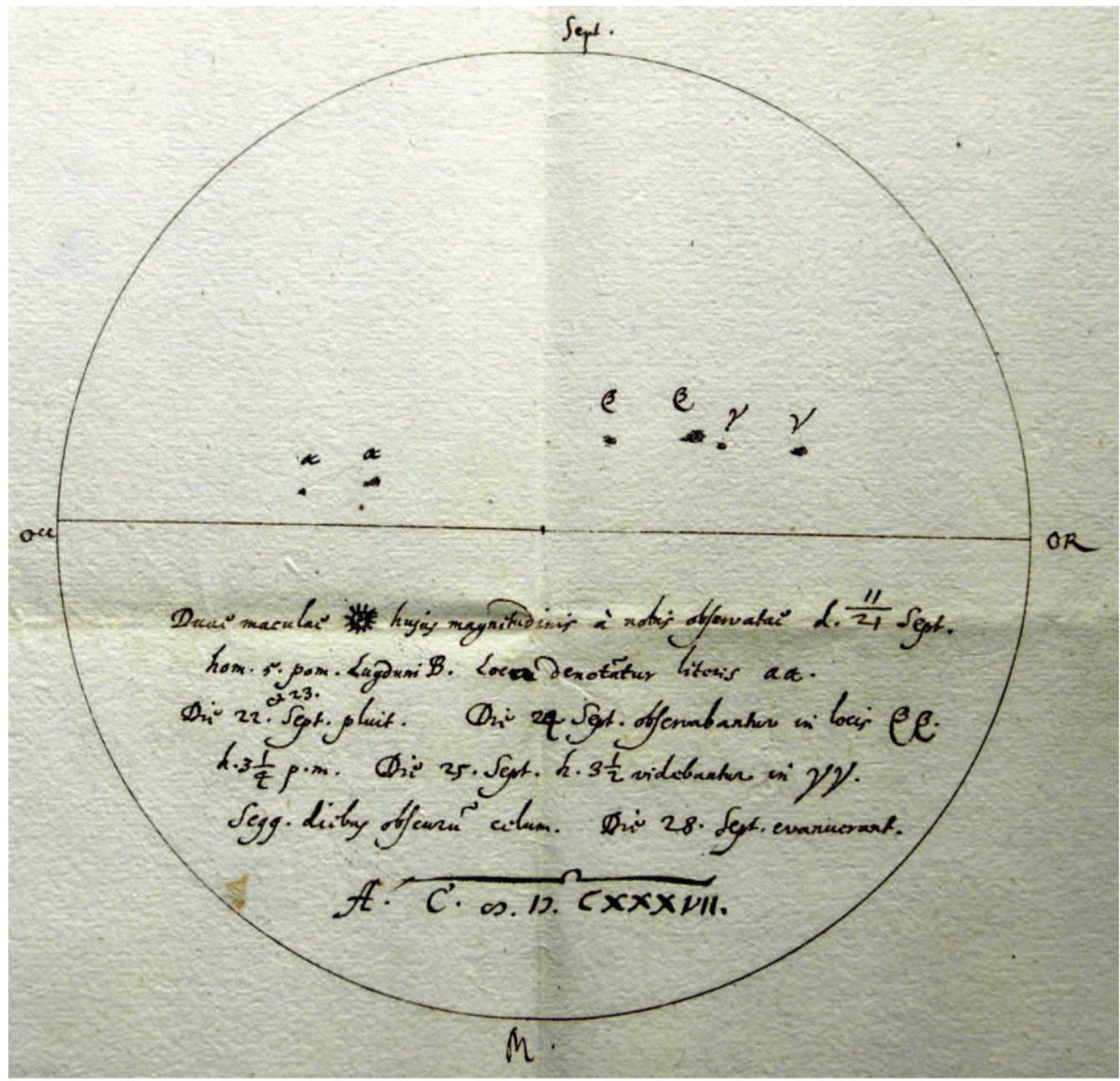

Figure 6. Example of the solar disk drawing by Marcgraft depicting sunspots from 1637 September 21 to 25 .

Toulmonde (1997), Pap et al. (2001), and Rozelot (2001). Since the 18th century, the values of solar radius rapidly converged towards the current value. Ribes et al. (1987) suggested the existence of a larger Sun during Maunder minimum. This claim was criticized by O'Dell \& Van Helden (1987), who argued that the error in the measurements made by the Paris observers was larger than Ribes et al. (1987) had assumed.

During the 17th and 18th centuries, meridian lines in cathedrals in Bologna, Rome, Florence, and Paris served as solar observatories making careful and continuous study of the Sun possible (Heilbron 1999). The earliest of these observatories was in the cathedral of San Petronio in Bologna. The heliometer of San Petronio consists of two separate pieces. One piece lies on the floor; it is a perfectly horizontal rod running due north for about $67 \mathrm{~m}$ from a spot under one of the side chapels to the front door of the church. The other part is a small hole $(2.5 \mathrm{~cm}$ in diameter $)$ in a horizontal metal plate fixed in the roof of the chapel. The hole is permanently open so as to give free access to solar rays around noon throughout the year. For 80 years (1655-1736), some scientists and 

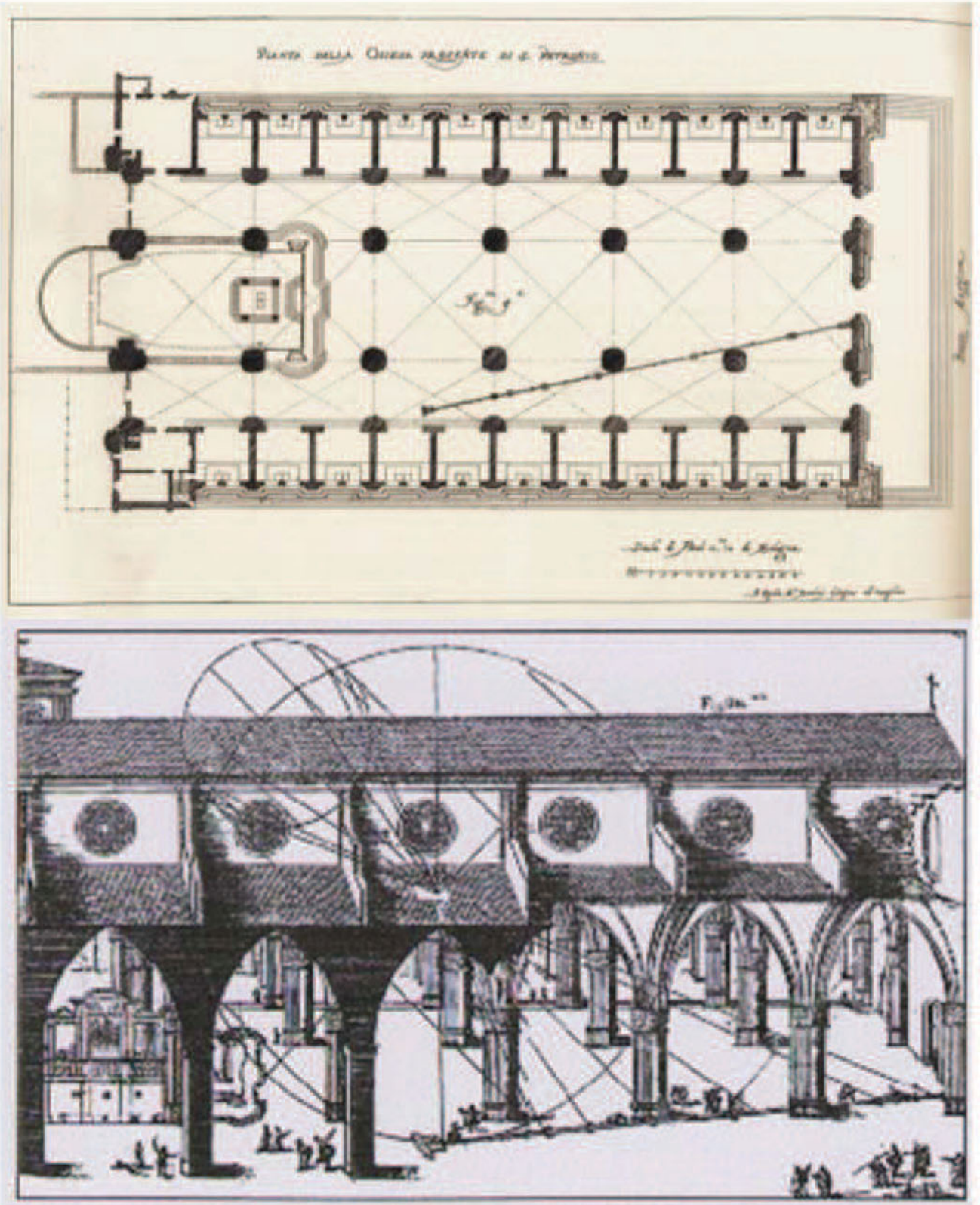

Figure 7. Old schemes of San Petronio Cathedral in Bologna (Heilbron, 1999).

clerics observed the Sun, filling about 300 pages in the register published by Eustachio Manfredi (1736). Each entry includes a description of the weather, the distances of the Sun's limbs from the vertex corrected for the penumbra, and the apparent diameter of the sun, all given to seconds of arc.

Vaquero \& Vázquez (2009), see Figure 5.10 in page 227, compiled all measurements of solar radius, in arcminutes, made in San Petronio from 1655 to 1736. This time series is 


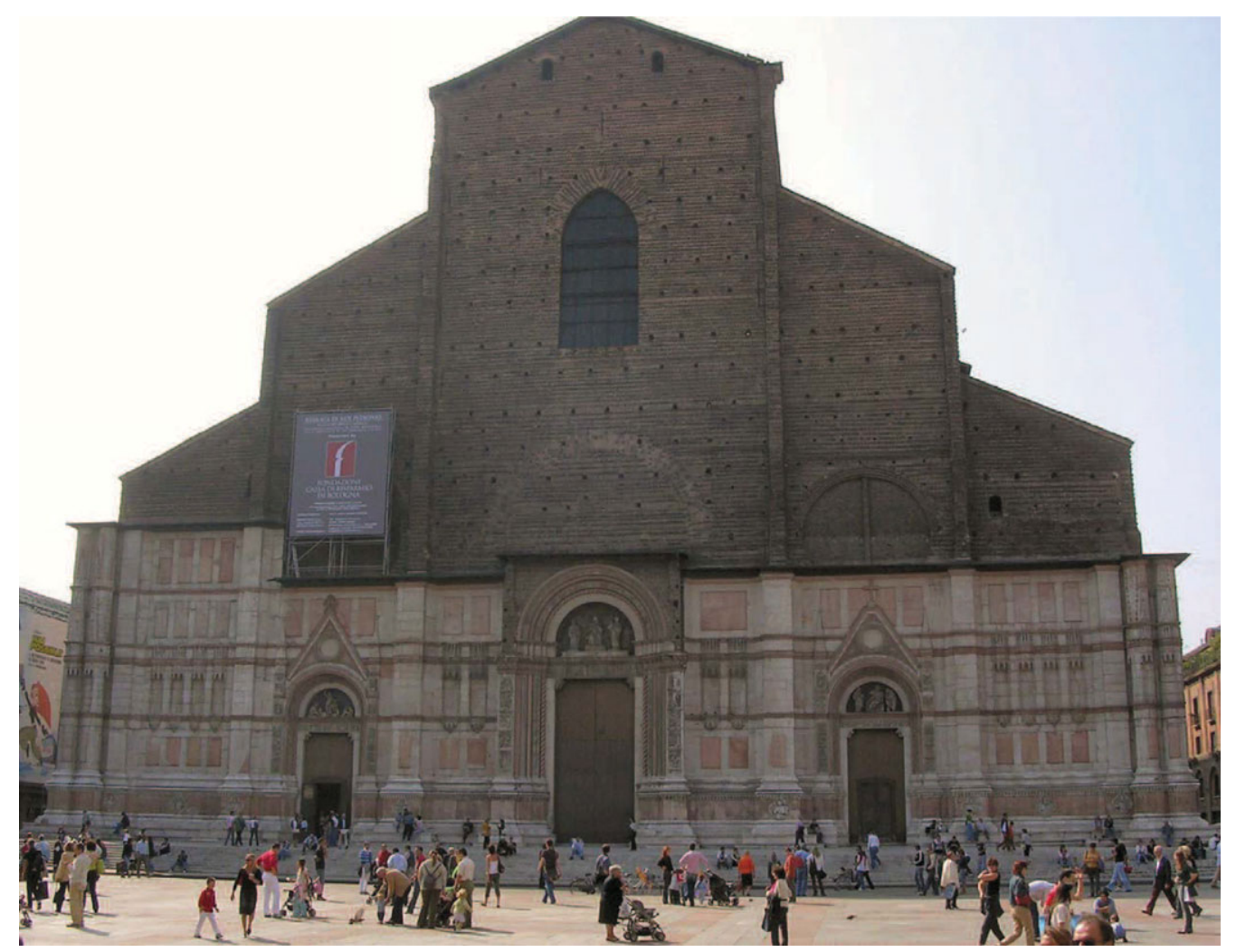

Figure 8. The Basilica di San Petronio in Bologna (picture by Paolo Carboni).

very noisy and it is not easy to obtain a clear conclusion. However, one can see clearly the annual variation of the apparent solar radius in this record in Figure 9, where only the period 1695-1705 is represented. This dataset require further studies.

\section{Conclusion}

Vaquero \& Vázquez (2009) have shown that solar Grand Minima of the last millennium are recorded in documentary solar proxies as naked-eye sunspot and auroral records. The well-known minima of Oort, Wolf, Spoerer, and Maunder stand out sharply in both records. Moreover, revised sunspot data prior to the Maunder minimum lead to the revisited observational scenario of a Grand minimum of solar activity. The new data dramatically change the magnitude of the sunspot cycle just before the Maunder minimum implying a possibly gradual onset of the minimum with reduced activity started two cycles before it (Vaquero et al. 2011).

Any information on the state of the Sun in past times is interesting because the Sun is not a laboratory experiment that can be controlled. Of course, there are useful data to study past solar activity preserved in libraries and archives. 


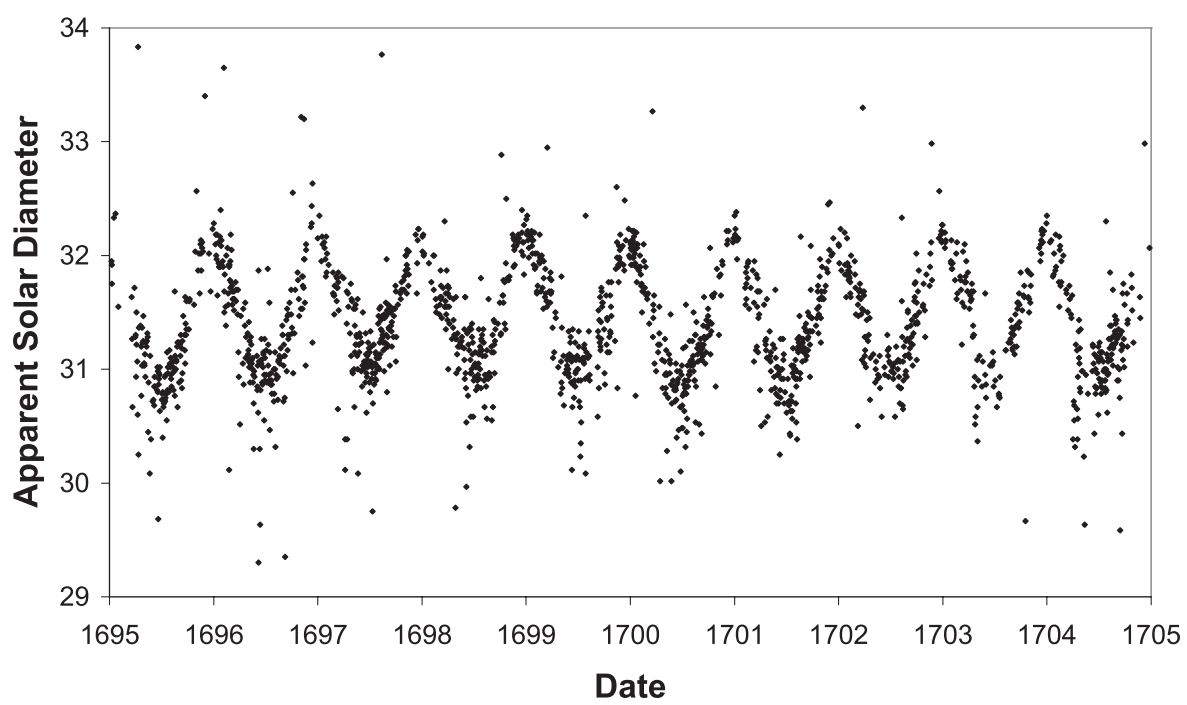

Figure 9. Measurements of solar radius, in arcminutes, made in San Petronio from 1695 to 1705 .

\section{Acknowledgements}

The author is grateful to I.G. Usoskin for his valuable comments. Support from the Junta de Extremadura and Ministerio de Ciencia e Innovacin of the Spanish Government (AYA2008-04864/AYA and AYA2011-25945) is gratefully acknowledged.

\section{References}

Akasofu, S.-I. 2007, Exploring the Secrets of the Aurora (Springer, 2nd Edition)

Boller, W. 1898, Gerlands Beiträge zur Geophysik, 3, 56, 550

Bone, N. 2007, Observing and Recording Nature's Spectacular Light Show (Springer, Patrick Moore's Practical Astronomy Series)

Brekke, A. \& Eggeland, A. 1994, The Northern Lights. Their Heritage and Science (Reidel)

Burland, C. A. 1958, In: Proceedings of the 32 International Congress of Americanist (Copenhague), 326-330

Casas, R., Vaquero, J. M., \& Vázquez, M. 2006, Solar Phys. 234, 379

Eather, R. H. 1980, Majestic Lights (Washington: American Geophysical Union)

Eddy, J. A. 1976, Science 192, 1189

Eddy, J. A. 1983, Solar Phys. 89, 195

Eddy, J. A., Stephenson, F. R., \& Yau, K. C. 1989, Q. J. R. Astron. Soc. 30, 65

Flammarion, C. 1873, L'Atmosphère, (Paris: Librairie Hachete et Cia.)

Fritz, H. 1873, Verzeichniss Beobachteter Polarlichter, (Wien: C. Gerold's Sohn)

Hameed, S. \& Gong, G. 1991, Solar Phys. 139, 409

Heilbron, J. L. 2001, The sun in the church: cathedrals as solar observatories (Harvard: Harvard University Press)

Hoyt, D. V. \& Schatten, K. H. 1998, Solar Phys. 179, 189

Krivsky, L. 1985, Bull. Astron. Inst. Czechosl., 36, 60

Krivsky, L. \& Pejml, K. 1988, Solar Activity, Aurorae and Climate in Central Europe in the last 1000 Years (Publications of the Astronomical Institute of the Czechoslovak Academy of Sciences, Publication No. 75)

Lee, E. H., Ahn, Y. S., Yang, H. J., \& Chen, K. Y. 2004, Solar Phys., 224, 373

Link, F. 1962, Geofysikalni Sbornik, 173, 297

Link, F. 1964, Geofysikalni Sbornik, 212, 501 
Malville, J. M. \& Singh, R. P. B. 1995, Vistas in Astronomy, 39, 431

Manfredi, E. 1736, De Gnomone Meridiano Bononiensi ad Divi Petronii (Bononiae: Laeli a Vulpa)

Miyahara, H., Sokoloff, D., \& Usoskin, I. G. 2006, The solar cycle at the Maunder minimum epoch. In: Ip, W.-H., Duldig, M. (Eds.), Advances in Geosciences (Singapore: World Scientific), pp. 120

Moore, S. 2003, J. Br. Astron. Assoc., 113(5), 305

Nagovitsyn, Yu. A. 2001, Geomagn. Aeron. 41, 711

O'Dell, C. R. \& Van Helden, A. 1987, Nature 330, 629

Pap, J., Rozelot, J. P., Godier, S., \& Varadi, F. 2001, Astron. Astrophys. 372, 1005

Ribes, E., Ribes, J. C., \& Barthalot, R. 1987, Nature 330, 629

Ribes, J. C. \& Nesme-Ribes, E. 1993, Astron. Astrophys. 276, 549

Rozelot, J. P. 2001, J. Atmos. Terr. Phys. 63, 375

Schaefer, B. E. 1991, Q. J. R. Astron. Soc., 32, 35

Schaefer, B. E. 1993, Astrophys. J., 411, 909

Scuderi, L. A. 1990, Q. J. R. Astron. Soc. 31, 109

Silverman, S. M. 1992, Reviews of Geophysics 30, 333

Solanki, S. K., Usoskin, I. G., Kromer, B., Schüssler, M., \& Beer, J. 2004, Nature, 431, 1084

Soon, W. W.-H. \& Yaskell, S. H. 2003, Maunder Minimum and the Variable Sun Earth Connections (Singapore: World Scientific Printers)

Stephenson, F. R. \& Willis, D. M. 1999, Astron. Geophys., 40, 21

Toulmonde, M. 1997, Astron. Astrophys. 325, 1174

Usoskin, I. G., Mursula, K., \& Kovaltsov, G. A. 2001, J. Geophys. Res. 106(A8), 16039

Usoskin, I. G. 2008, Living Rev. Solar Phys., 5, 3 (http://www.livingreviews.org/lrsp-2008-3)

Vaquero, J. M. \& Gallego, M. C. 2002, Solar Phys., 206, 209

Vaquero, J. M., Gallego, M. C., \& García, J. A. 2002, Geophys. Res. Lett., 29, 1997

Vaquero, J. M. 2004, Solar Phys., 223, 283

Vaquero, J. M. 2007, Adv. Spa. Res. 40, 929

Vaquero, J. M. \& Vázquez, M. 2009, The Sun Recorded Through History (New York: Springer)

Vaquero, J. M., Gallego, M. C., Usoskin, I. G., \& Kovaltsov, G. A. 2011, ApJL 731, L24

Willis, D. M., Easterbrook, M. G., \& Stephenson, F. R. 1980, Nature 287, 617

Willis, D. M., Davda, V. N., \& Stephenson, F. R. 1996, Q. J. R. Astron. Soc. 37, 189

Wittmann, A. D. \& Xu, Z. T. 1987, Astron. Astrophys. Suppl. Series, 70, 83

Yau, K. K. C. \& Stephenson, F. R. 1988, Q. J. R. Astron. Soc., 29, 175

\section{Discussion}

NADEZHDA Zolotova: Is it correct that due to uncertainties in the solar images, it is not possible to identify not only the latitude but even the hemisphere where the sunspots are?

José VAquero: In general, my answer is "yes". However, we have also some detailed and well oriented old sunspot drawings. 